\title{
The Al Doping Effect on Epitaxial (In,Mn)As Dilute Magnetic Semiconductors Prepared by Ion Implantation and Pulsed Laser Melting
}

\author{
Ye Yuan ${ }^{1,2, *}$, Yufang Xie ${ }^{2}$, Ning Yuan ${ }^{2}$, Mao Wang ${ }^{2}$, René Heller ${ }^{2}$, Ulrich Kentsch ${ }^{2}$, Tianrui Zhai ${ }^{3}$ (i) \\ and Xiaolei Wang ${ }^{3, *}$ \\ 1 Songshan Lake Materials Laboratory, Dongguan 523808, China \\ 2 Helmholtz-Zentrum Dresden-Rossendorf, Institute of Ion Beam Physics and Materials Research, \\ Bautzner Landstrasse 400, 01328 Dresden, Germany; y.xie@hzdr.de (Y.X.); n.yuan@hzdr.de (N.Y.); \\ m.wang@hzdr.de (M.W.); r.heller@hzdr.de (R.H.); U.Kentsch@hzdr.de (U.K.) \\ 3 College of Physics and Optoelectronics, Faculty of Science, Beijing University of Technology, \\ Beijing 100124, China; trzhai@bjut.edu.cn \\ * Correspondence: yuanye@sslab.org.cn (Y.Y.); xiaoleiwang@bjut.edu.cn (X.W.)
}

check for

updates

Citation: Yuan, Y.; Xie, Y.; Yuan, N.; Wang, M.; Heller, R.; Kentsch, U.; Zhai, T.; Wang, X. The Al Doping Effect on Epitaxial (In,Mn)As Dilute Magnetic Semiconductors Prepared by Ion Implantation and Pulsed Laser Melting. Materials 2021, 14, 4138. https://doi.org/10.3390/ma14154138

Academic Editors: Francesco Biccari and Thomas Niendorf

Received: 9 June 2021

Accepted: 23 July 2021

Published: 25 July 2021

Publisher's Note: MDPI stays neutral with regard to jurisdictional claims in published maps and institutional affiliations.

Copyright: (c) 2021 by the authors. Licensee MDPI, Basel, Switzerland. This article is an open access article distributed under the terms and conditions of the Creative Commons Attribution (CC BY) license (https:/ / creativecommons.org/licenses/by/ $4.0 /)$.

\begin{abstract}
One of the most attractive characteristics of diluted ferromagnetic semiconductors is the possibility to modulate their electronic and ferromagnetic properties, coupled by itinerant holes through various means. A prominent example is the modification of Curie temperature and magnetic anisotropy by ion implantation and pulsed laser melting in III-V diluted magnetic semiconductors. In this study, to the best of our knowledge, we performed, for the first time, the co-doping of (In,Mn)As diluted magnetic semiconductors by Al by co-implantation subsequently combined with a pulsed laser annealing technique. Additionally, the structural and magnetic properties were systematically investigated by gradually raising the $\mathrm{Al}$ implantation fluence. Unexpectedly, under a well-preserved epitaxial structure, all samples presented weaken Curie temperature, magnetization, as well as uniaxial magnetic anisotropies when more aluminum was involved. Such a phenomenon is probably due to enhanced carrier localization introduced by $\mathrm{Al}$ or the suppression of substitutional Mn atoms.
\end{abstract}

Keywords: magnetic semiconductors; ion implantation; co-doping; magnetic properties

\section{Introduction}

III-Mn-V diluted ferromagnetic semiconductors (DFSs) have received a great deal of attention because of their enormous potential for spintronic application [1,2] due to their characteristic that electronic and ferromagnetic properties are coupled by itinerant holes [3-8]. However, because the ultra-low solubility of Mn in III-V semiconductors is significantly (normally several orders of magnitude) below the threshold value of appearing ferromagnetism, the preparation of epitaxial DFS film is extremely challenged. Even for the most canonical DFS (Ga,Mn)As, mostly low-temperature molecular beam epitaxy (LT-MBE) has been employed for its epitaxial film preparation $[4,7,8]$, which significantly prohibited the development of DFSs. Moreover, such an obstacle threatens the most conventional technique of semiconductor modification, i.e., co-doping which is productive to present new physics [9-14]. However, ion implantation followed by the pulsed laser melting method provides an alternative solution to this problem due to its second non-equilibrium grown essence, and therefore, it has been used in several hyper-doping cases in which some are even impossible to approach by LT-MBE [15-17]. Thus, it is important to explore the cohyper-doping effect in various DFSs by ion implantation and pulsed laser melting (PLM).

According to the $p$ - $d$ Zenner model [3,5], Curie temperature and magnetization of DFS are strongly commensurate with $p$ - $d$ hybridization, and such $p$ - $d$ coupling is, in principle, more intensive between $\mathrm{Al}$ and As than between $\mathrm{Ga}$ and As. Accordingly, $(\mathrm{Al}, \mathrm{Mn}) \mathrm{As}$ is theoretically expected to exhibit higher $T_{\mathrm{C}}$ than $(\mathrm{Ga}, \mathrm{Mn}) \mathrm{As}$ at the same 
Mn concentration and hole density [3]. Unfortunately, such an expectation has not been subsequently observed in LT-MBE grown Al-doped $(\mathrm{Ga}, \mathrm{Mn})$ As sample $[13,18]$, in which only magnetic anisotropy switching happens, and such behavior is explained by the enhanced hole localization introduced by $\mathrm{Al}$ doping. It is worth while noting that the absence of an apparent increase in $T_{\mathrm{C}}$ is probably due to the stronger $p$ - $d$ hybridization between $\mathrm{Al}$ and As, which enables the system to be more insulating and also reduces magnetization. Therefore, it seems that the $T_{\mathrm{C}}$ modification is a trade-off between $p-d$ coupling enhancement (increase $T_{\mathrm{C}}$ ) and $p-d$ coupling enhancement induced by carrier localization (decrease $T_{\mathrm{C}}$ ) in various DFS candidates [13,18-20]. On the one hand, it is important to study the Al-doped (In,Mn)As, since the $p$ - $d$ coupling between $\mathrm{Al}$ and As is much stronger than that between In and As [3,5,21]; however, InAs presents a higher hole mobility [22,23], and accordingly, a high $T_{C}$ is expected in Al-doped (In,Mn)As. On the other hand, due to the challenged preparation of epitaxial (In,Mn)As DFSs, studies on co-doping effect in (In,Mn)As are limited and new phenomena should be investigated.

In this study, Al-co-doped (In,Mn)As samples were realized by ion implantation combined with a pulsed laser melting technique, and by gradually increasing the $\mathrm{Al} \mathrm{im-}$ plantation fluences, the $\mathrm{Al}$ doping concentration was accordingly raised. According to the results of Rutherford backscattering spectrometry and ion channeling (RBS/C), all (In,Mn)As and Al-co-doped (In,Mn)As films display epitaxial structure, which is comparable with the virgin InAs wafer. Upon raising the Al content, magnetization and $T_{C}$ are both apparently reduced together with weakened out-of-plane uniaxial magnetic anisotropy, which is probably due to the enhanced localization or reduced substitutional Mn atoms. Similar phenomena have been observed in our previous studies on co-doped $(\mathrm{Ga}, \mathrm{Mn}) \mathrm{As}$ and $(\mathrm{Ga}, \mathrm{Mn}) \mathrm{P}$ samples.

\section{Materials and Methods}

The Al-doped (In,Mn)As samples were prepared by ion implantation combined with the PLM technique. Before the Al implantation, all InAs substrates were implanted with $\mathrm{Mn}$ ions to achieve the $\mathrm{Mn}$ doping. Mn and $\mathrm{Al}$ implantation were both performed at an angle of $7^{\circ}$ to avoid channeling effect. For Mn doping, the implantation fluence was set as $2.4 \times 10^{16} / \mathrm{cm}^{2}$, and the implantation energy was $100 \mathrm{keV}$ at room temperature. Afterwards, $\mathrm{Al}$ implantation was carried out on post $\mathrm{Mn}$ implanted samples with fluences of $1.7 \times 10^{16} / \mathrm{cm}^{2}$ and $3.4 \times 10^{16} / \mathrm{cm}^{2}$ at an energy value of $60 \mathrm{keV}$ at room temperature, and the samples were referred to as InMnAlAs-4 and InMnAlAs-8, respectively. One Mn implanted sample was selected as the reference for a comparison, i.e., InMnAlAs0 . After implantation, the doped region became amorphous, and thus recrystallization was necessary. For the annealing process, a UV pulsed laser with nanosecond pulse was employed for the recrystallization. The pulsed laser treatment with a $28 \mathrm{~ns}$ duration was performed in air atmosphere, and an energy of $0.2 \mathrm{~J} / \mathrm{cm}^{2}$ was selected, which is the optimal annealing condition for InAs recrystallization [21,24]. The wavelength of the pulse laser is $308 \mathrm{~nm}$. Magnetic properties were explored by a Superconducting Quantum Interference Device vibrating sample magnetometer (SQUID-VSM, Quantum Design, US) equipped with a low field option. For the temperature dependent thermo-remanent magnetization (TRM) measurement, first, the sample was cooled from 300 to $5 \mathrm{~K}$ under a $5 \mathrm{kOe}$ magnetic field for magnetic saturation. Afterwards, the field was totally removed by magnet reset operation, and then the warm process started; meanwhile, the data collection began.

\section{Results and Discussion}

\subsection{SRIM Calculation}

Figure 1 displays the results of $\mathrm{Mn}$ and $\mathrm{Al}$ distribution in the InAs matrix by the stopping and range of ions in matter (SRIM) calculations [25]. As shown in the figure, both $\mathrm{Mn}$ and $\mathrm{Al}$ present a Gaussian distribution at a depth of $200 \mathrm{~nm}$. Interestingly, even the implanting energy of $\mathrm{Mn}(100 \mathrm{keV})$ is much larger than that of $\mathrm{Al}(60 \mathrm{keV})$, and the $\mathrm{Mn}$ maximal peak appears at a shallower depth $(54 \mathrm{~nm})$ than that of $\mathrm{Al}(67 \mathrm{~nm})$, due to the 
different stopping abilities of InAs lattice to these two elements. According to the SRIM simulation, the longitudinal straggling for $\mathrm{Mn}$ and $\mathrm{Zn}$ in the InAs matrix is 37.5 and $53 \mathrm{~nm}$, respectively, thus, the thickness of the $\mathrm{Mn}$ - and $\mathrm{Zn}$-doped regions can be treated as 75 and $106 \mathrm{~nm}$ by $2 \Delta \mathrm{R}_{\mathrm{P}}$, respectively. The overlap of the doped regions indicates a valid co-doping effect. When the atomic density of InAs is considered, the atomic ratio of implanted atoms to indium atoms is easily obtained. As a result, the maximal Mn concentration is $14.7 \%$ when the implanting fluence is $2.4 \times 10^{16} / \mathrm{cm}^{2}$, and the average doping concentration is around $12 \%$. However, according to our previous study [24], the only-Mn-doped sample exhibited an average Mn concentration of $8.7 \%$ in the doped region, due to the diffusion of Mn caused by pulsed laser-induced liquid phase epitaxy. For Al implantation, the average concentrations are calculated as $6 \%$ and $12 \%$, respectively, when the implantation fluences are selected as $1.7 \times 10^{16} / \mathrm{cm}^{2}$ and $3.4 \times 10^{16} / \mathrm{cm}^{2}$.

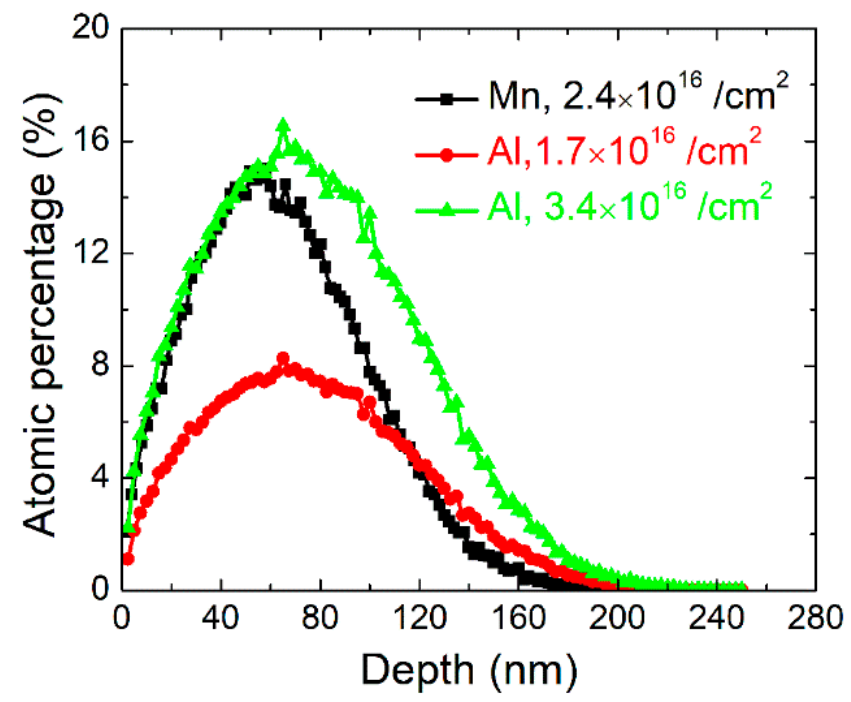

Figure 1. SRIM calculation of implanted $\mathrm{Mn}$ and $\mathrm{Al}$ distribution in InAs matrix.

\subsection{Structure of All Co-Doped Samples}

For recrystallizing as-implanted samples, a pulse ( $28 \mathrm{~ns})$ UV laser was employed to activate the implanted dopants. During the pulsed laser melting treatment, most laser energy is absorbed by the amorphized implanted region. The absorbed energy is so high that the whole implanted region begins to melt, whereas the InAs substrate remains at room temperature. As a result, the huge temperature gradient drives ultra-fast recrystallization at a speed of several $\mathrm{m} / \mathrm{s}$ and the so-called liquid epitaxy process happens. Due to the ultra-fast growth process, implanted $\mathrm{Mn}$ and $\mathrm{Al}$ atoms have no time to perform long-range diffusion and are driven into the matrix lattice.

To reveal the structure of Mn- and Al-co-doped and Mn-doped InAs samples, Raman spectroscopy was used. The spectra are shown in Figure 2. All samples present two obvious peaks at wavenumbers of 225 and $233 \mathrm{~cm}^{-1}$. For the InMnAlAs- 0 sample, a broadened signal and a sharp LO signal are both observed, indicating that both vibration modes are detectable from the [001] direction. However, according to the selection rule of the zinc-blende structure, only the longitudinal optical (LO) phonon mode is allowed in the backscattering configuration, whereas the transverse optical (TO) phonon mode is forbidden. Therefore, it is not possible that the peak at $225 \mathrm{~cm}^{-1}$ is original from the TO mode. Actually, due to the presence of itinerant holes inside the Mn-doped region, part of the LO signals transfer into coupled plasmon-LO-phonon mode (CPLOM) which is present at a wavelength of $225 \mathrm{~cm}^{-1}$ between LO vibration and itinerant holes [26]. However, the introduction of $\mathrm{Al}$ atoms into the (In,Mn)As and the enhanced carry localization by $\mathrm{Al}$ involvement largely eliminate coupled contribution by free holes $[27,28]$. Therefore, the CPLOM signal is largely suppressed in the InMnAlAs- 4 sample as compared with in the 
InMnAlAs-0 sample. However, the continuous increase of $\mathrm{Al}$ concentration increases the lattice disorder in the matrix, which leads to an intensive broadening of the vibration peak.

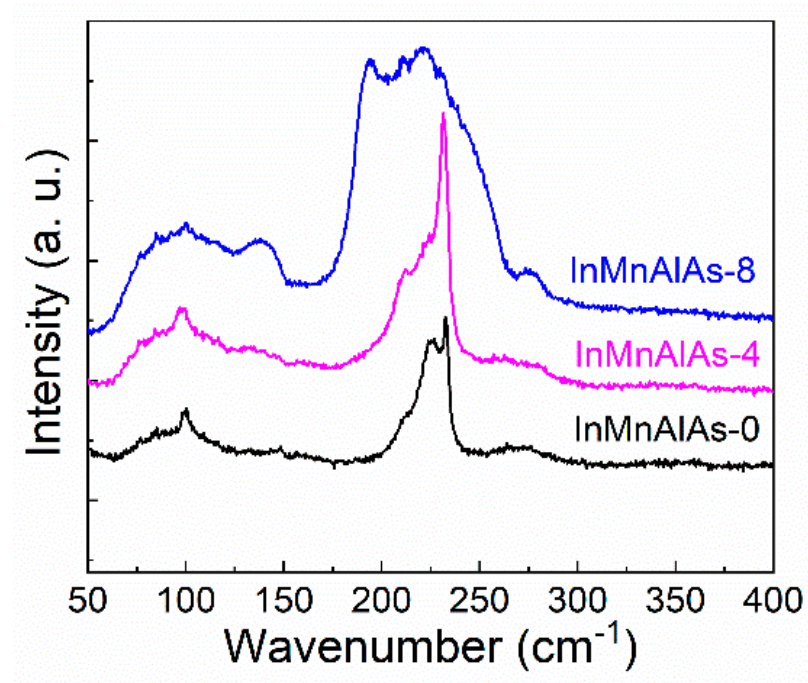

Figure 2. Raman spectra of samples InMnAlAs-0 (black); InMnAlAs-4 pink); InMnAlAs-8 (blue).

In addition to Raman microscopy, the structure of all co-doped samples together with the reference (In,Mn)As and virgin InAs samples are investigated by RBS/C. THE results are shown in Figure 3. According to the random spectra, indium and arsenic signals are both pronounced; however, the $\mathrm{Mn}$ and $\mathrm{Al}$ signals are much weaker, even invisible, due to a low concentration of only several percentages and the overlapping with the strong signal from In and As. After normalization, it is possible to evaluate the crystalline quality by the ratio of backscattering yields obtained under the channeling conditions and the random beam incidence, which is defined as $\chi_{\min }$. As shown in Figure 3, upon increasing $\mathrm{Al}$ doping density, the $\chi_{\mathrm{min}}$ only reveals a slight increase, from $9.8 \%$-via $11.0 \%, 11.2 \%$, and finally to $13.3 \%$-indicating that crystallization deviates slightly from the InAs lattice, which is caused by $\mathrm{Mn}$ and $\mathrm{Al}$ doping. However, the epitaxial nature of the co-doped films persists, which excludes the contribution from amorphization or polycrystaliziation to the latter discussed manipulation of the magnetic properties.

\subsection{Magnetic Properties}

During the gradually increasing $\mathrm{Al}$ implantation fluences, the evolvement of magnetic properties is investigated, and one ( $\mathrm{In}, \mathrm{Mn}) \mathrm{As}$ without $\mathrm{Al}$ doping is present as a reference. Figure $4 \mathrm{a}$ shows the magnetic field dependent magnetization after subtracting the diamagnetic signal from InAs substrates. The InMnAlAs-0 sample presents a typical ferromagnetic feature, i.e., highly square-like hysteresis loop with a low coercivity field (107 Oe) and a magnetization of $\sim 23 \mathrm{emu} / \mathrm{cm}^{3}$. According to Figure $4 \mathrm{~b}$, the sample shows a high $T_{C}$ of $80 \mathrm{~K}$ where the magnetization vanishes [24]. Moreover, the $M-T$ curve shows a concave-like shape, and this is reminiscent of mean-field theory approximation $[5,24,29]$. The magnetic field and temperature dependent magnetization both unambiguously prove the ferromagnetic nature of our virgin ( $\mathrm{In}, \mathrm{Mn})$ As. Unexpectedly, the $\mathrm{Al}$ doping directly results in reducing magnetization and enhancing coercivity; the saturation magnetization gradually decreases from 23.5 , via 19.8 , finally to $15.7 \mathrm{emu} / \mathrm{cm}^{3}$; meanwhile, the coercivity contrarily rises from 107 via 674 to 993 Oe. Additionally, the thermo-remanent magnetization at $5 \mathrm{~K}$ decreases from 23.0, via 20.3 , finally to $14.1 \mathrm{emu} / \mathrm{cm}^{3}$. Actually, the suppression of magnetization and Curie temperature induced by $\mathrm{Al}$ doping has been observed in a series of $(\mathrm{Ga}, \mathrm{Mn})$ As samples [13]; in a study by [3], there were two different mechanisms proposed: (i) part of Mn atoms are driven into the interstitial sites; and (ii) the localization of carriers is enhanced by $\mathrm{Al}$ alloying due to stronger $p-d$ exchange coupling However, in the low $\mathrm{Al}$ concentration doped region $(<20 \%)$, the first function worked dominantly, 
and a similar phenomenon appeared in $\mathrm{Zn}$-doped $(\mathrm{Ga}, \mathrm{Mn}) \mathrm{As}$ according to our previous research [14]. This is the most probable explanation for the Al-doped (In,Mn)As case, since, for the highest $\mathrm{Al}$ concentration doped sample, it is observed that in addition to the reduction in Curie temperature, the $M-T$ curve deviates from the mean-field approximation, and such a phenomenon is in good agreement with a decrease in substitutional $\mathrm{Mn}$ concentration [24]. Unfortunately, it is extremely difficult to examine the effect of enhanced localization $[24,25]$ in our sample, because of the conducting InAs substrate.

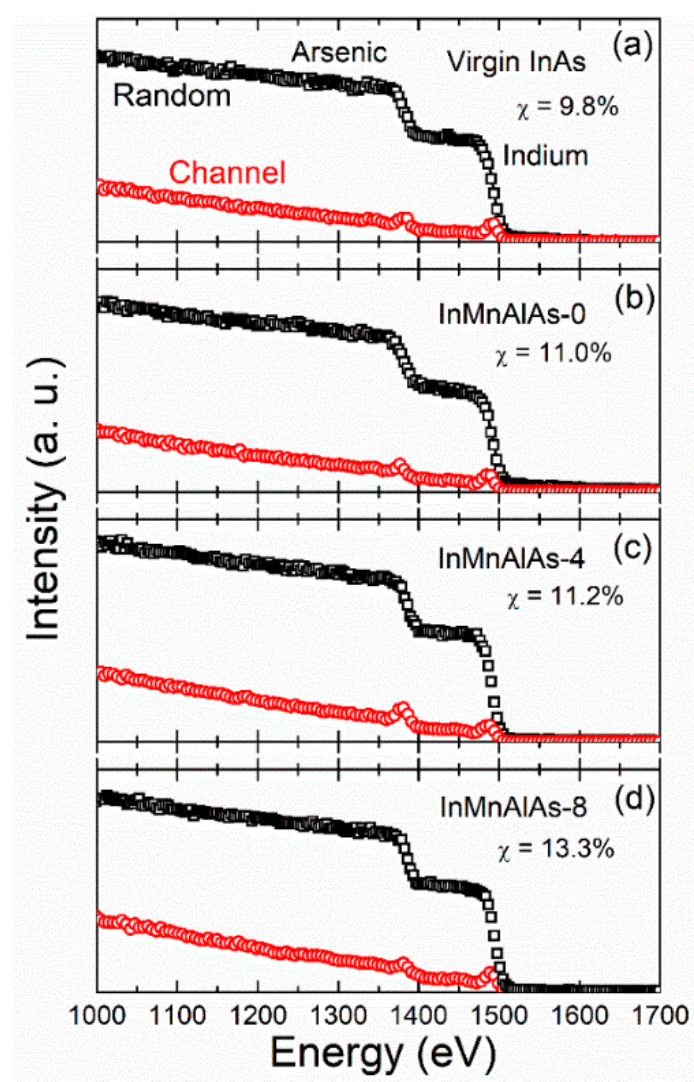

Figure 3. The RBS random (open squares) and channeling (open circles) spectra of samples: (a) virgin InAs; (b) InMnAlAs-0; (c) InMnAlAs-4; (d) InMnAlAs-8.

According to an XMCD (X-ray magnetic circular dichroism) study on Al-doped (Ga,Mn)As by Edmonds et al. [27], the doping of aluminum modulates the strain state and further leads to switching of the uniaxial magnetic anisotropy. Thus, it is worthwhile investigating the changing of magnetic anisotropy upon increasing the concentration of aluminum in our (In,Mn)As samples. The results are shown in Figure 5. However, in our samples, although all samples present typical uniaxial magnetic anisotropy with an out-of-plane magnetic easy axis, the anisotropy feature presents a changing behavior with increasing $\mathrm{Al}$ doping concentration. As shown in Figure 5a, the InMnAlAs-0 sample exhibits a specific perpendicular uniaxial magnetic anisotropy; the $M-H$ curve is highly square-like when the magnetic field is applied along the out-of-plane direction, while a much higher saturation field $(2.5 \mathrm{kOe})$ and lower remanent magnetization are both observed along the in-plane direction. Such a phenomenon duplicates the same characteristics in previous (In,Mn)As prepared by both ion implantation and LT-MBE [6,24]. 

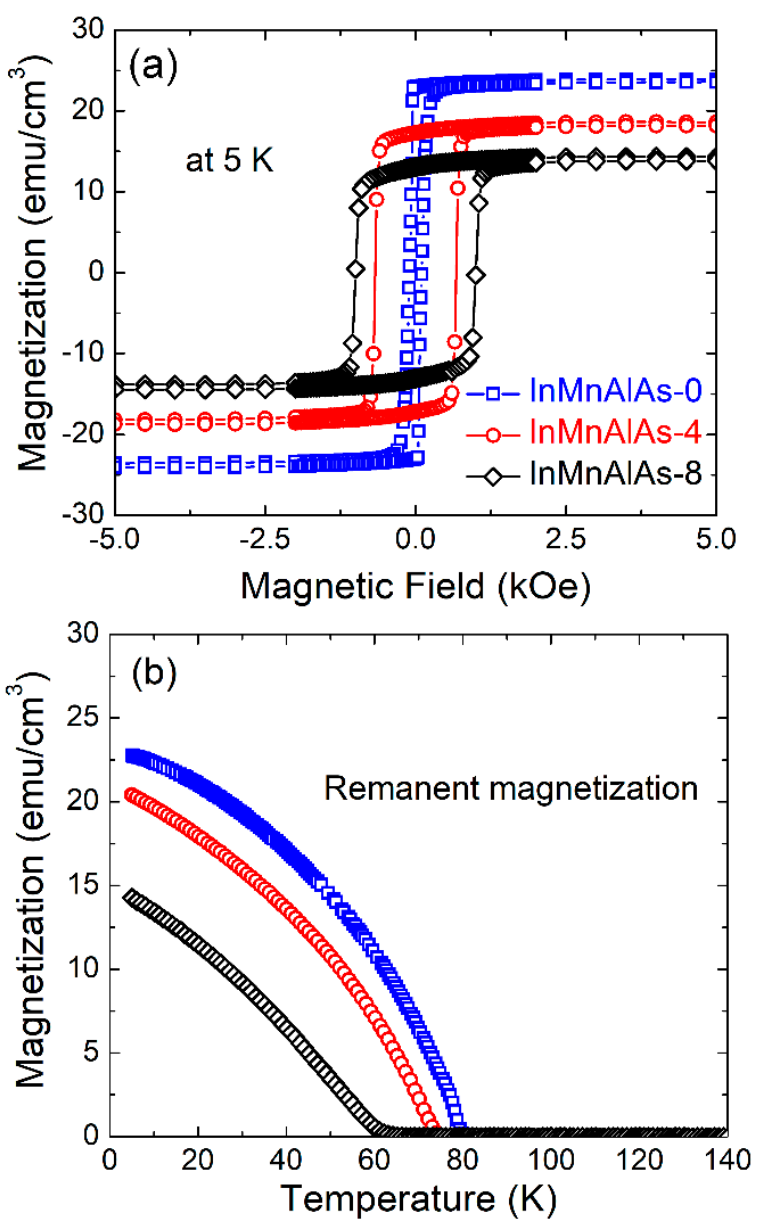

Figure 4. (a) Magnetic field dependent magnetization of the InMnAlAs-0 (squares), InMnAlAs-4 (circles), and InMnAlAs-8 (diamonds) samples, at $5 \mathrm{~K}$, when the magnetic field is applied along the out-of-plane direction; (b) temperature dependent remanent magnetization at zero field of three samples.

However, when the doped $\mathrm{Al}$ concentration is increased, the $M-H$ hysteresis loops of the out-of-plane direction gradually change from a square shape, while the $M-H$ curves along the in-plane direction start to open a loop. Such a transform can be quantitatively evaluated by the anisotropy constant. Calculated from the difference of integral area of $M-H$ loops between out-of-plane and in-plane directions, the anisotropy constants are $2.71 \times 10^{4}, 2.13 \times 10^{4}$, and $1.60 \times 10^{4} \mathrm{erg} / \mathrm{cm}^{3}$ for the InMnAlAs-0, InMnAlAs-4, and InMnAlAs-8 samples, respectively. There is a decline in the anisotropy constants; however, there is no change in the uniaxial magnetic anisotropy as He irradiated GaMnAsP [30]. For a comparison, the magnetic properties of all samples are listed in Table 1.

Table 1. Al implantation fluences, remanent magnetization, Curie temperature, coercivity, and anisotropy constant for the InMnAlAs-0, InMnAlAs-4, and andInMnAlAs-8 samples.

\begin{tabular}{|c|c|c|c|c|c|}
\hline Sample No. & Al Imp. Flu. & $\begin{array}{c}M_{\mathrm{R}} \\
\left(\mathrm{emu} / \mathrm{cm}^{3}\right)\end{array}$ & $T_{\mathrm{C}}(\mathrm{K})$ & $H_{\mathrm{C}}(\mathrm{Oe})$ & $K\left(10^{4} \mathrm{erg} / \mathrm{cm}^{3}\right)$ \\
\hline InMnAlAs-0 & 0 & 22.7 & 80 & 107 & 2.71 \\
\hline InMnAlAs-4 & $1.7 \times 10^{16}$ & 20.3 & 72 & 674 & 2.13 \\
\hline InMnAlAs-8 & $3.4 \times 10^{16}$ & 14.3 & 60 & 993 & 1.60 \\
\hline
\end{tabular}



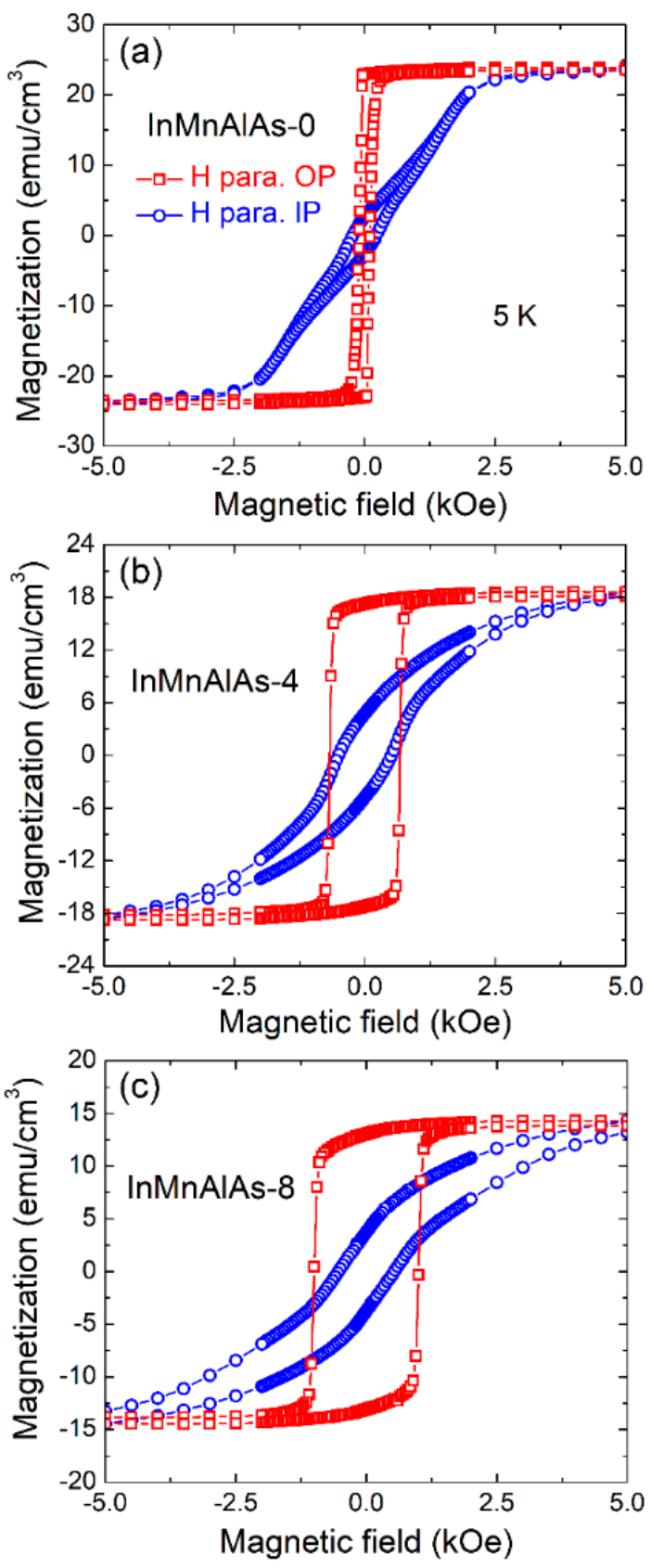

Figure 5. The magnetic field dependent magnetization of samples: (a) InMnAlAs-0; (b) InMnAlAs-4; (c) InMnAlAs-8, when the magnetic field is applied parallel (circles) and perpendicular (squares) with the plane.

\section{Conclusions}

In summary, we firstly report the co-doping effect of (In,Mn)As by Al through coimplantation combined with a pulsed laser melting technique. Upon increasing the Al concentration by raising implantation fluences, all doped samples present well-preserved epitaxial structures, and the magnetic properties are tuned. The Curie temperature and magnetization are reduced together with modifications of the anisotropy constants. Unexpectedly, the above-mentioned phenomena are not induced by the amorphization, which is confirmed by the RBS channeling spectra, and it is possible to explain the phenomenon by the enhanced carrier localization or the decreased substitutional Mn atoms introduced by aluminum incorporation. 
Author Contributions: Y.Y. and X.W. conceived the project; Y.Y. and Y.X. prepared the samples; Y.Y. and N.Y. carried out the magnetic measurements; M.W., R.H., and U.K. completed the RBS and ion implantation operation; X.W., T.Z. and Y.Y. analyzed the data and prepared the manuscript. All authors contributed to the discussion of the results. All authors have read and agreed to the published version of the manuscript.

Funding: This research was funded by the Key-Area Research and Development Program of Guangdong Province (2019B121204004), National Natural Science Foundation of China (NSFC, grant no. 12074018), as well as the Basic and Application Basic Research Foundation of Guangdong Province (grant no. 2020A1515110891). The support from the Ion Beam Center (IBC) at HZDR is highly appreciated.

Institutional Review Board Statement: Not applicable.

Informed Consent Statement: Not applicable.

Data Availability Statement: The data presented in this study are available in the article.

Conflicts of Interest: The authors declare no conflict of interest.

\section{References}

1. Kosuke, T.; Duc, A.L.; Chiba, T.; Tomohiro, K.; Chiba, D.; Masaaki, T. Giant gate-controlled proximity magnetoresistance in semiconductor-based ferromagnetic-non-magnetic bilayers. Nat. Phys. 2019, 15, 1134-1139.

2. Dietl, T.; Bonanni, A.; Ohno, H. Families of magnetic semiconductors-An overview. J. Semicond. 2019, 40, 080301. [CrossRef]

3. Dietl, T.; Ohno, H.; Matsukura, F.; Cibert, J.; Ferrand, D. Zener Model Description of Ferromagnetism in Zinc-Blende Magnetic Semiconductors. Science 2000, 287, 1019-1022. [CrossRef] [PubMed]

4. Dietl, T.; Ohno, H. Dilute ferromagnetic semiconductors: Physics and spintronic structures. Rev. Mod. Phys. 2014, 86, 187-251. [CrossRef]

5. Dietl, T.; Ohno, H.; Matsukura, F. Hole-mediated ferromagnetism in tetrahedrally coordinated semiconductors. Phys. Rev. B 2001, 63, 195205. [CrossRef]

6. Ohno, H.; Chiba, D.; Matsukura, F.; Omiya, T.; Abe, E.; Dietl, T.; Ohno, Y.; Ohtani, K. Electric-field control of ferromagnetism. Nature 2000, 408, 944-946. [CrossRef]

7. Chiba, D.; Sawicki, M.; Nishitani, Y.; Nakatani, Y.; Matsukura, F.; Ohno, H. Magnetization vector manipulation by electric fields. Nature 2008, 455, 515-518. [CrossRef]

8. Chiba, D.; Yamanouchi, M.; Matsukura, F.; Ohno, H. Electrical Manipulation of Magnetization Reversal in a Ferromagnetic Semiconductor. Science 2003, 301, 943-945. [CrossRef] [PubMed]

9. Ferrand, D.; Cibert, J.; Bourgognon, C.; Tatarenko, S.; Wasiela, A.; Fishman, G.; Bonanni, A.; Sitter, H.; Kolesnik, S.; Jaroszyski, J.; et al. Carrier-induced ferromagnetic interactions in p-doped $\mathrm{Zn}_{1-\mathrm{x}} \mathrm{Mn}_{\mathrm{x}}$ Te epilayers. J. Cryst. Growth 2000, 214, 387-390. [CrossRef]

10. Ferrand, D.; Cibert, J.; Wasiela, A.; Bourgognon, C.; Tatarenko, S.; Fishman, G.; Andrearczyk, T.; Jaroszynski, J.; Kolesnik, S.; Dietl, T.; et al. Carrier-induced ferromagnetism in p- $\mathrm{Zn}_{1-\mathrm{x}} \mathrm{Mn}_{\mathrm{x}}$ Te. Phys. Rev. B 2001, 63, 085201. [CrossRef]

11. Casiraghi, A.; Rushforth, A.W.; Wang, M.; Farley, N.R.S.; Wadley, P.; Hall, J.L.; Staddon, C.R.; Edmonds, K.W.; Campion, R.P.; Foxon, C.T.; et al. Tuning perpendicular magnetic anisotropy in $(\mathrm{Ga}, \mathrm{Mn})(\mathrm{As}, \mathrm{P})$ by thermal annealing. Appl. Phys. Lett. 2010, 97, 122504. [CrossRef]

12. Rushforth, A.W.; Wang, M.; Farley, N.R.S.; Campion, R.P.; Edmonds, K.W.; Staddon, C.R.; Foxon, C.T.; Gallagher, B.L. Molecular beam epitaxy grown $(\mathrm{Ga}, \mathrm{Mn})(\mathrm{As}, \mathrm{P})$ with perpendicular to plane magnetic easy axis. J. Appl. Phys. 2009, 104, 073908. [CrossRef]

13. Rushforth, A.W.; Farley, N.R.S.; Campion, R.P.; Edmonds, K.W.; Staddon, C.R.; Foxon, C.T.; Gallagher, B.L.; Yu, K.M. Compositional dependence of ferromagnetism in (Al,Ga,Mn)As magnetic semiconductors. Phys. Rev. B 2008, 78, 085209. [CrossRef]

14. Xu, C.; Zhang, C.; Wang, M.; Xie, Y.; Hübner, R.; Heller, R.; Yuan, Y.; Helm, M.; Zhang, X.; Zhou, S. p-type codoping effect in (Ga,Mn)As: Mn lattice location versus magnetic properties. Phys. Rev. Mater. 2019, 3, 084604. [CrossRef]

15. Stone, P.R.; Alberi, K.; Tardif, S.K.Z.; Beeman, J.W.; Yu, K.M.; Walukiewicz, W.; Dubon, O.D. Metal-Insulator Transition by Isovalent Anion Substitution in $\mathrm{Ga}_{1-x} \mathrm{Mn}_{x}$ As: Implications to Ferromagnetism. Phys. Rev. Lett. 2008, 101, 087203. [CrossRef]

16. Stone, P.R.; Bihler, C.; Kraus, M.; Scarpulla, M.A.; Beeman, J.W.; Yu, K.M.; Brandt, M.S.; Dubon, O.D. Compensation-dependent in-plane magnetization reversal processes in $\mathrm{Ga}_{1-x} \mathrm{Mn}_{\mathrm{x}} \mathrm{PyS}_{1-\mathrm{y}}$. Phys. Rev. B 2008, 78, 21442. [CrossRef]

17. Xu, C.; Yuan, Y.; Wang, M.; Hentschel, H.; Bottger, R.; Helm, M.; Zhou, S. p-type co-doping effect of (Ga,Mn)P: Magnetic and magneto-transport properties. J. Magn. Magn. Mater. 2018, 459, 102-105. [CrossRef]

18. Mašek, J.; Kudrnovský, J.; Máca, F.; Sinova, J.; MacDonald, A.H.; Campion, R.P.; Gallagher, B.L.; Jungwirth, T. Mn-doped Ga(As,P) and (Al,Ga)As ferromagnetic semiconductors: Electronic structure calculations. Phys. Rev. B 2007, 75, 045202. [CrossRef]

19. Wang, X.L.; Wang, H.L.; Ma, J.L.; Zhao, X.P.; Zhao, J.H. Efficiently rotating the magnetization vector in a magnetic semiconductor via organic molecules. ACS Appl. Mater. Interfaces 2019, 11, 6615. [CrossRef] [PubMed] 
20. Wang, X.L.; Wang, H.L.; Pan, D.; Keiper, T.; Li, L.X.; Yu, X.Z.; Lu, J.; Lochner, E.; von Molnár, S.; Xiong, P.; et al. Robust manipulation of magnetism in dilute magnetic semiconductor (Ga,Mn)As by organic molecules. Adv. Mater. 2015, $27,8043$. [CrossRef]

21. Yuan, Y.; Xu, C.; Hübner, R.; Jakiela, R.; Böttger, R.; Helm, M.; Sawicki, M.; Dietl, T.; Zhou, S. Interplay between localization and magnetism in (Ga,Mn)As and (In,Mn)As. Phys. Rev. Mater. 2017, 1, 054401. [CrossRef]

22. Oiwa, A.; Endo, A.; Katsumoto, S.; Iye, Y.; Ohno, H.; Munekata, H. Magnetic and transport properties of the ferromagnetic semiconductor heterostructures (In,Mn)As/(Ga,Al) Sb. Phys. Rev. B 1999, 59, 5826. [CrossRef]

23. Munekata, H. Anomalous Hall effect in III-V-based magnetic semiconductor heterostructures. Mater. Sci. Eng. B 1995, 31, 151-156. [CrossRef]

24. Yuan, Y.; Wang, Y.; Gao, K.; Khalid, M.; Wu, C.; Zhang, W.; Munnik, F.; Weschke, E.; Baehtz, C.; Skorupa, W.; et al. High Curie temperature and perpendicular magnetic anisotropy in homoepitaxial InMnAs films. J. Phys. D Appl. Phys. 2015, 48, 235002. [CrossRef]

25. Ziegler, J.F.; Ziegler, M.D.; Biersack, J.P. SRIM-The stopping and range of ions in matter. Nucl. Instrum. Methods Phys. Res. Sect. B 2010, 268, 1818. [CrossRef]

26. Yuan, Y.; Hübner, R.; Liu, F.; Sawicki, M.; Gordan, O.; Salvan, G.; Zahn, D.R.T.; Banerjee, D.; Baehtz, C.; Helm, M.; et al. Ferromagnetic Mn-Implanted GaP: Microstructures vs. Magnetic Properties. ACS Appl. Mater. Interfaces 2016, 8, $3912-3918$. [CrossRef]

27. Edmonds, K.W.; van der Laan, G.; Farley, N.R.S.; Arenholz, E.; Campion, R.P.; Foxon, C.T.; Gallagher, B.L. Strain dependence of the Mn anisotropy in ferromagnetic semiconductors observed by x-ray magnetic circular dichroism. Phys. Rev. B 2008, 77, 113205. [CrossRef]

28. Van der Laan, G.; Edmonds, K.W.; Arenholz, E.; Farley, N.R.S.; Gallagher, B.L. Valence state model of strain-dpendent Mn L2,3 x-ray magnetic circular dichroism from ferromagnetic semiconductors. Phys. Rev. B 2010, 81, 214422. [CrossRef]

29. Arrott, A.; Noakes, J.E. Approximate Equation of State For Nickel Near its Critical Temperature. Phys. Rev. Lett. 1967, 19, 786. [CrossRef]

30. Yuan, Y.; Amarouche, T.; Xu, C.; Rushforth, A.; Böttger, R.; Edmonds, K.; Campion, R.; Gallagher, B.; Helm, M.; von Bardeleben, H.J.; et al. Switching the uniaxial magnetic anisotropy by ion irradiation induced compensation. J. Phys. D Appl. Phys. 2018, 51, 145001. [CrossRef] 